\title{
Approximate Nash Region of the Gaussian Interference Channel with Noisy Output Feedback
}

\author{
Victor Quintero, Samir M. Perlaza, Jean-Marie Gorce, and H. Vincent Poor
}

\begin{abstract}
In this paper, an achievable $\eta$-Nash equilibrium $(\eta$ $\mathrm{NE}$ ) region for the two-user Gaussian interference channel with noisy channel-output feedback is presented for all $\eta \geqslant 1$. This result is obtained in the scenario in which each transmitterreceiver pair chooses its own transmit-receive configuration in order to maximize its own individual information transmission rate. At an $\eta$-NE, any unilateral deviation by either of the pairs does not increase the corresponding individual rate by more than $\eta$ bits per channel use.
\end{abstract}

Index Terms-Gaussian Interference Channel, Noisy channeloutput feedback, $\eta$-Nash equilibrium region.

\section{INTRODUCTION}

The interference channel (IC) is one of the simplest yet insightful multi-user channels in network information theory. An important class of ICs is the two-user Gaussian interference channel (GIC) in which there exist two point-to-point links subject to mutual interference and independent Gaussian noise sources. In this model, each output signal is a noisy version of the sum of the two transmitted signals affected by the corresponding channel gains. The analysis of this channel can be made considering two general scenarios: (1) a centralized scenario in which the entire network is controlled by a central entity that configures both transmitter-receiver pairs; and (2) a decentralized scenario in which each transmitter-receiver pair autonomously configures its transmission-reception parameters. In the former, the fundamental limits are characterized by the capacity region, which is approximated to within a fixed number of bits in [1] for the case without feedback; in [2] for the case with perfect channel-output feedback; and in [3] and [4] for the case with noisy channel-output feedback. In the latter, the fundamental limits are characterized by the $\eta$-Nash equilibrium ( $\eta$-NE) region. The $\eta$-NE of the GIC is approximated in the cases without feedback and with perfect channel-output feedback in [5] and [6], respectively.

Victor Quintero is with the Department of Telecommunications, Universidad del Cauca, 19003, Popayán, Cauca, Colombia. (vflorez@unicauca.edu.co).

Samir M. Perlaza and Jean-Marie Gorce are with the laboratoire CITI (a joint laboratory between the Université de Lyon, INRIA, and INSA de Lyon). 6 Avenue des Arts, F-69621, Villeurbanne, France. (\{samir.perlaza, jean-marie.gorce\}@inria.fr).

H. Vincent Poor and Samir M. Perlaza are with the Department of Electrical Engineering at Princeton University, Princeton, NJ 08544 USA. (poor@princeton.edu).

This research was supported in part by the European Commission under Marie Skłodowska-Curie Individual Fellowship No. 659316; in part by the INSA Lyon - SPIE ICS chair on the Internet of Things; in part by the Administrative Department of Science, Technology, and Innovation of Colombia (Colciencias), fellowship No. 617-2013; and in part by the U. S. National Science Foundation under Grants CNS-1702808 and ECCS-1647198.

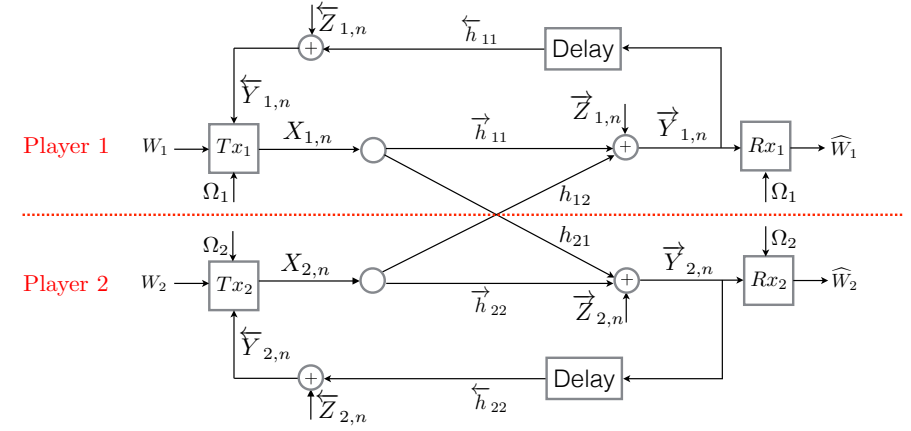

Fig. 1. Two-User Decentralized Gaussian interference channel with noisy channel-output feedback at channel use $n$.

In this paper the $\eta$-NE region of the GIC is studied assuming that there exists a noisy feedback link from each receiver to its corresponding transmitter. The $\eta$-NE region is approximated by two regions for all $\eta \geqslant 1$ : a region for which an equilibrium transmit-receive configuration is presented for each of the information rate pairs (an achievable region); and a region for which any information rate pair that is outside of this region cannot be an $\eta$-NE (impossibility region). The focus of this paper is on the achievable region.

The results presented in this paper are a generalization of the results presented in [5] and [6], and they are obtained thanks to the analysis of linear deterministic approximations in [7] and [8].

\section{DeCentralized Gaussian Interference ChanNels With NoIsy Channel-OUtPut FeEdBaCK}

Consider the two-user decentralized Gaussian interference channel with noisy channel-output feedback (D-GIC-NOF) depicted in Figure 11. Transmitter $i$, with $i \in\{1,2\}$, communicates with receiver $i$ subject to the interference produced by transmitter $j$, with $j \in\{1,2\} \backslash\{i\}$. There are two independent and uniformly distributed messages, $W_{i} \in \mathcal{W}_{i}$, with $\mathcal{W}_{i}=$ $\left\{1,2, \ldots,\left\lfloor 2^{N_{i} R_{i}}\right\rfloor\right\}$, where $N_{i}$ denotes the fixed block-length in channel uses and $R_{i}$ the information transmission rate in bits per channel use. At each block, transmitter $i$ sends the codeword $\boldsymbol{X}_{i}=\left(X_{i, 1}, X_{i, 2}, \ldots, X_{i, N_{i}}\right)^{\top} \in \mathcal{C}_{i} \subseteq \mathbb{R}^{N_{i}}$, where $\mathcal{C}_{i}$ is the codebook of transmitter $i$. The channel coefficient from transmitter $j$ to receiver $i$ is denoted by $h_{i j}$; the channel coefficient from transmitter $i$ to receiver $i$ is denoted by $\vec{h}_{i i}$; and the channel coefficient from channel-output $i$ to transmitter $i$ is denoted by $\overleftarrow{h}_{i i}$. All channel coefficients are assumed to be non-negative real numbers. At a given channel 
use $n \in\{1,2, \ldots, N\}$, with

$$
N=\max \left(N_{1}, N_{2}\right)
$$

the channel output at receiver $i$ is denoted by $\vec{Y}_{i, n}$. During channel use $n$, the input-output relation of the channel model is given by

$$
\vec{Y}_{i, n}=\vec{h}_{i i} X_{i, n}+h_{i j} X_{j, n}+\vec{Z}_{i, n},
$$

where $X_{i, n}=0$ for all $n$ such that $N \geqslant n>N_{i}$ and $\vec{Z}_{i, n}$ is a real Gaussian random variable with zero mean and unit variance that represents the noise at the input of receiver $i$. Let $d>0$ be the finite feedback delay measured in channel uses. At the end of channel use $n$, transmitter $i$ observes $\overleftarrow{Y}_{i, n}$, which consists of a scaled and noisy version of $\vec{Y}_{i, n-d}$. More specifically,

$$
\overleftarrow{Y}_{i, n}= \begin{cases}\overleftarrow{Z}_{i, n} & \text { for } n \in\{1,2, \ldots, d\} \\ \overleftarrow{h}_{i i} \vec{Y}_{i, n-d}+\overleftarrow{Z}_{i, n}, & \text { for } n \in\{d+1, d+2, \ldots, N\}\end{cases}
$$

where $\overleftarrow{Z}_{i, n}$ is a real Gaussian random variable with zero mean and unit variance that represents the noise in the feedback link of transmitter-receiver pair $i$. The random variables $\vec{Z}_{i, n}$ and $\overleftarrow{Z}_{i, n}$ are assumed to be independent. In the following, without loss of generality, the feedback delay is assumed to be one channel use, i.e., $d=1$. The encoder of transmitter $i$ is defined by a set of deterministic functions $f_{i, 1}^{(N)}, f_{i, 2}^{(N)}, \ldots, f_{i, N_{i}}^{(N)}$, with $f_{i, 1}^{(N)}: \mathcal{W}_{i} \times \mathbb{N} \rightarrow \mathcal{X}_{i}$ and for all $n \in\left\{2,3, \ldots, N_{i}\right\}, f_{i, n}^{(N)}:$ $\mathcal{W}_{i} \times \mathbb{N} \times \mathbb{R}^{n-1} \rightarrow \mathcal{X}_{i}$, such that

$$
\begin{aligned}
& X_{i, 1}=f_{i, 1}^{(N)}\left(W_{i}, \Omega_{i}\right), \text { and } \\
& X_{i, n}=f_{i, n}^{(N)}\left(W_{i}, \Omega_{i}, \overleftarrow{Y}_{i, 1}, \overleftarrow{Y}_{i, 2}, \ldots, \overleftarrow{Y}_{i, n-1}\right)
\end{aligned}
$$

where $\Omega_{i}$ is an additional index randomly generated. The index $\Omega_{i}$ is assumed to be known by both transmitter $i$ and receiver $i$, while unknown by transmitter $j$ and receiver $j$.

The components of the input vector $\boldsymbol{X}_{i}$ are real numbers subject to an average power constraint

$$
\frac{1}{N_{i}} \sum_{n=1}^{N_{i}} \mathbb{E}_{X_{i, n}}\left[X_{i, n}^{2}\right] \leq 1
$$

The decoder of receiver $i$ is defined by a deterministic function $\psi_{i}^{(N)}: \mathbb{N} \times \mathbb{R}^{N} \rightarrow \mathcal{W}_{i}$. At the end of the communication, receiver $i$ uses the vector $\left(\vec{Y}_{i, 1}, \vec{Y}_{i, 2}, \ldots, \vec{Y}_{i, N}\right)$ and the index $\Omega_{i}$ to obtain an estimate

$$
\widehat{W}_{i}=\psi_{i}^{(N)}\left(\Omega_{i}, \vec{Y}_{i, 1}, \vec{Y}_{i, 2}, \ldots, \vec{Y}_{i, N}\right),
$$

A transmit-receive configuration for transmitter-receiver pair $i$, denoted by $s_{i}$, can be described in terms of the blocklength $N_{i}$, the rate $R_{i}$, the codebook $\mathcal{C}_{i}$, the encoding functions $f_{i, 1}^{(N)}, f_{i, 2}^{(N)}, \ldots, f_{i, N_{i}}^{(N)}$, and the decoding function $\psi_{i}^{(N)}$, etc. The average error probability at decoder $i$ given the configurations $s_{1}$ and $s_{2}$, denoted by $p_{i}\left(s_{1}, s_{2}\right)$, is given by

$$
p_{i}\left(s_{1}, s_{2}\right)=\operatorname{Pr}\left[W_{i} \neq \widehat{W}_{i}\right] .
$$

Within this context, a rate pair $\left(R_{1}, R_{2}\right) \in \mathbb{R}_{+}^{2}$ is said to be achievable if it complies with the following definition.
Definition 1 (Achievable Rate Pairs): $A$ rate pair $\left(R_{1}, R_{2}\right) \in \mathbb{R}_{+}^{2}$ is achievable if there exists at least one pair of configurations $\left(s_{1}, s_{2}\right)$ such that the decoding bit error probabilities $p_{1}\left(s_{1}, s_{2}\right)$ and $p_{2}\left(s_{1}, s_{2}\right)$ can be made arbitrarily small by letting the block-lengths $N_{1}$ and $N_{2}$ grow to infinity.

The aim of transmitter $i$ is to autonomously choose its transmit-receive configuration $s_{i}$ in order to maximize its achievable rate $R_{i}$. Note that the rate achieved by transmitterreceiver $i$ depends on both configurations $s_{1}$ and $s_{2}$ due to mutual interference. This reveals the competitive interaction between both links in the decentralized interference channel. The fundamental limits of the two-user D-GIC-NOF in Figure 1 can be described by six parameters: $\overrightarrow{\mathrm{SNR}}_{i}, \overleftarrow{\mathrm{SNR}}_{i}$, and $\mathrm{INR}_{i j}$, with $i \in\{1,2\}$ and $j \in\{1,2\} \backslash\{i\}$, which are defined as follows:

$$
\begin{aligned}
& \overrightarrow{\mathrm{SNR}}_{i} \triangleq \vec{h}_{i i}^{2} \\
& \mathrm{INR}_{i j} \triangleq h_{i j}^{2} \text { and } \\
& \overleftarrow{\mathrm{SNR}}_{i} \triangleq \overleftarrow{h}_{i i}^{2}\left(\vec{h}_{i i}^{2}+2 \vec{h}_{i i} h_{i j}+h_{i j}^{2}+1\right) .
\end{aligned}
$$

The analysis presented in this paper focuses exclusively on the case in which $\mathrm{INR}_{i j}>1$ for all $i \in\{1,2\}$ and $j \in\{1,2\} \backslash\{i\}$. The reason for exclusively considering this case follows from the fact that when $\mathrm{INR}_{i j} \leqslant 1$, the transmitter-receiver pair $i$ is impaired mainly by noise instead of interference. In this case, feedback does not bring a significant rate improvement. Denote by $\mathcal{C}$ the capacity region of the two-user GIC-NOF with fixed parameters $\overrightarrow{\mathrm{SNR}}_{1}, \overrightarrow{\mathrm{SNR}}_{2}$, $\mathrm{INR}_{12}, \mathrm{INR}_{21}, \overleftarrow{\mathrm{SNR}}_{1}$, and $\overleftarrow{\mathrm{SNR}}_{2}$. The achievable region $\mathcal{C}$ in [4, Theorem 2] and the converse region $\overline{\mathcal{C}}$ in [4, Theorem3] approximate the capacity region $\mathcal{C}$ to within 4.4 bits [4].

\section{GAME FORMULATION}

The competitive interaction between the two transmitterreceiver pairs in the interference channel can be modeled by the following game in normal-form:

$$
\mathcal{G}=\left(\mathcal{K},\left\{\mathcal{A}_{k}\right\}_{k \in \mathcal{K}},\left\{u_{k}\right\}_{k \in \mathcal{K}}\right) .
$$

The set $\mathcal{K}=\{1,2\}$ is the set of players, that is, the set of transmitter-receiver pairs. The sets $\mathcal{A}_{1}$ and $\mathcal{A}_{2}$ are the sets of actions of players 1 and 2, respectively. An action of a player $i \in \mathcal{K}$, which is denoted by $s_{i} \in \mathcal{A}_{i}$, is basically its transmitreceive configuration as described above. The utility function of player $i$ is $u_{i}: \mathcal{A}_{1} \times \mathcal{A}_{2} \rightarrow \mathbb{R}_{+}$and it is defined as the information rate of transmitter $i$,

$$
u_{i}\left(s_{1}, s_{2}\right)=\left\{\begin{array}{lll}
R_{i}, & \text { if } & p_{i}\left(s_{1}, s_{2}\right)<\epsilon \\
0, & \text { otherwise }
\end{array}\right.
$$

where $\epsilon>0$ is an arbitrarily small number. This game formulation for the case without feedback was first proposed in [9] and [10].

A class of transmit-receive configurations that are particularly important in the analysis of this game is referred to as the set of $\eta$-Nash equilibria ( $\eta$-NE), with $\eta>0$. This type of configurations satisfy the following definition. 


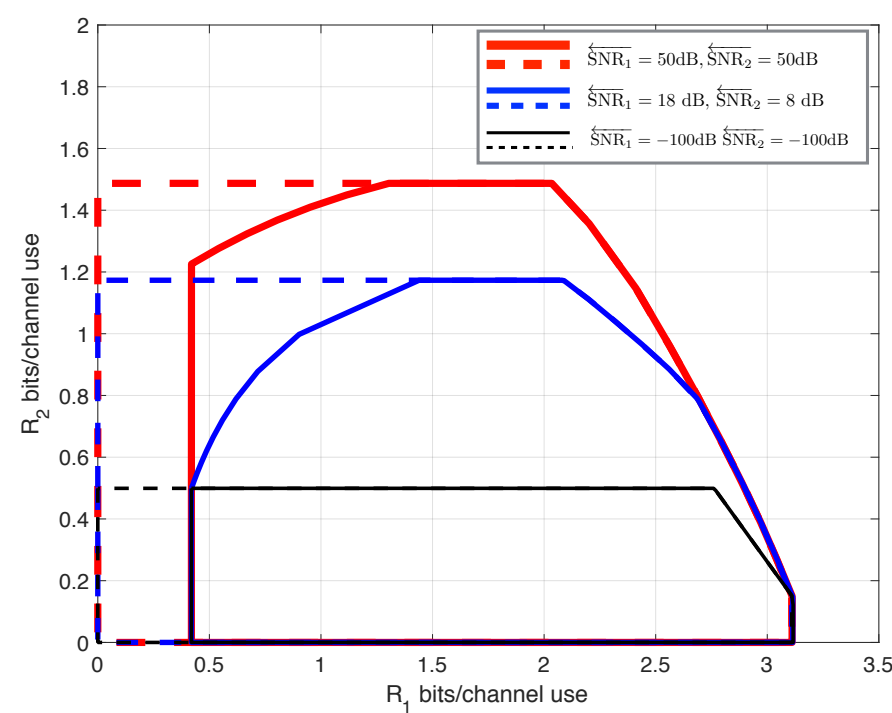

Fig. 2. Achievable capacity regions $\underline{\mathcal{C}}$ (dashed-lines) in [4 Theorem 2]

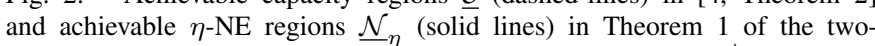
user GIC-NOF and two-user D-GIC-NOF with parameters $\overrightarrow{\mathrm{SNR}}_{1}=24 \mathrm{~dB}$, $\overrightarrow{\mathrm{SNR}}_{2}=3 \mathrm{~dB}, \mathrm{INR}_{12}=16 \mathrm{~dB}, \mathrm{INR}_{21}=9 \mathrm{~dB}, \overleftarrow{\mathrm{SNR}}_{1} \in\{-100,18,50\}$ $\mathrm{dB}, \overleftarrow{\mathrm{SNR}}_{2} \in\{-100,8,50\} \mathrm{dB}$ and $\eta=1$.

Definition 2 ( $\eta$-Nash equilibrium): Given a positive real $\eta$, an action profile $\left(s_{1}^{*}, s_{2}^{*}\right)$ is an $\eta$-Nash equilibrium $(N E)$ in the game $\mathcal{G}=\left(\mathcal{K},\left\{\mathcal{A}_{k}\right\}_{k \in \mathcal{K}},\left\{u_{k}\right\}_{k \in \mathcal{K}}\right)$, if for all $i \in \mathcal{K}$ and for all $s_{i} \in \mathcal{A}_{i}$, it follows that

$$
u_{i}\left(s_{i}, s_{j}^{*}\right) \leqslant u_{i}\left(s_{i}^{*}, s_{j}^{*}\right)+\eta \text {. }
$$

Let $\left(s_{1}^{*}, s_{2}^{*}\right)$ be an $\eta$-Nash equilibrium action profile. Then, none of the transmitters can increase its own transmission rate more than $\eta$ bits per channel use by changing its own transmit-receive configuration and keeping the average bit error probability arbitrarily close to zero. Note that for $\eta$ sufficiently large, from Definition 2, any pair of configurations can be an $\eta$-NE. Alternatively, for $\eta=0$, the definition of Nash equilibrium is obtained [11]. In this case, if a pair of configurations is a Nash equilibrium $(\eta=0)$, then each individual configuration is optimal with respect to each other. Hence, the interest is to describe the set of all possible $\eta$-NE rate pairs $\left(R_{1}, R_{2}\right)$ of the game in (11) with the smallest $\eta$ for which there exists at least one equilibrium configuration pair.

The set of rate pairs that can be achieved at an $\eta$-NE is known as the $\eta$-Nash equilibrium ( $\eta$-NE) region.

Definition 3 ( $\eta$-NE Region): Let $\eta>0$ be fixed. An achievable rate pair $\left(R_{1}, R_{2}\right)$ is said to be in the $\eta$-NE region of the game $\mathcal{G}=\left(\mathcal{K},\left\{\mathcal{A}_{k}\right\}_{k \in \mathcal{K}},\left\{u_{k}\right\}_{k \in \mathcal{K}}\right)$ if there exists a pair $\left(s_{1}^{*}, s_{2}^{*}\right) \in \mathcal{A}_{1} \times \mathcal{A}_{2}$ that is an $\eta$-NE and the following holds:

$$
u_{1}\left(s_{1}^{*}, s_{2}^{*}\right)=R_{1} \quad \text { and } \quad u_{2}\left(s_{1}^{*}, s_{2}^{*}\right)=R_{2} .
$$

The $\eta$-NE regions of the two-user GIC with and without perfect channel-output feedback have been approximated to within a constant number of bits in [5] and [6], respectively. The next section introduces a generalization of these results.

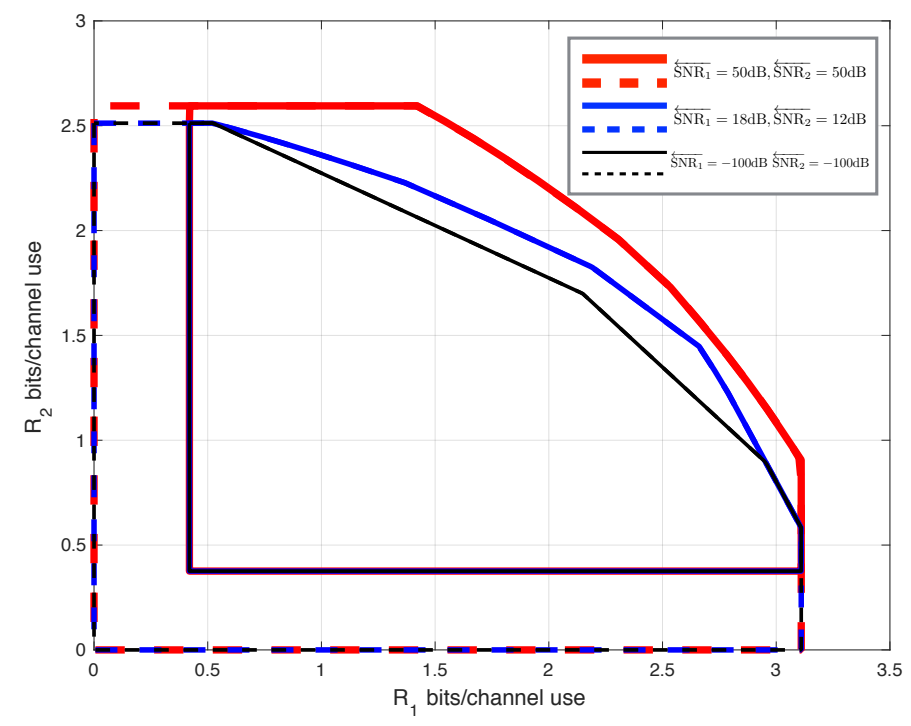

Fig. 3. Achievable capacity regions $\underline{\mathcal{C}}$ (dashed-lines) in [4 Theorem 2] and achievable $\eta$-NE regions $\underline{\mathcal{N}}_{\eta}$ (solid lines) in Theorem 11 of the twouser GIC-NOF and two-user D-GIC-NOF with parameters $\overrightarrow{S N R}_{1}=24$ $\mathrm{dB}, \overrightarrow{\mathrm{SNR}}_{2}=18 \mathrm{~dB}_{2} \mathrm{INR}_{12}=16 \mathrm{~dB}, \mathrm{INR}_{21}=10 \mathrm{~dB}, \overleftarrow{\mathrm{SNR}}_{1} \in$ $\{-100,18,50\} \mathrm{dB}, \overleftarrow{\mathrm{SNR}}_{2} \in\{-100,12,50\} \mathrm{dB}$ and $\eta=1$.

\section{Main Results}

\section{A. Achievable $\eta$-Nash Equilibrium Region}

Let the $\eta$-NE region (Definition 3) of the D-GIC-NOF be denoted by $\mathcal{N}_{\eta}$. This section introduces a region $\underline{\mathcal{N}}_{\eta} \subseteq \mathcal{N}_{\eta}$ that is achievable using a coding scheme that combines rate splitting [12], common randomness [5], [6], block Markov superposition coding [13] and backward decoding [14]. In the following, this coding scheme is referred to as randomized Han-Kobayashi scheme with noisy channel-output feedback (RHK-NOF). This coding scheme is presented in [8] and uses the same techniques of the schemes in [5] and [6]. Therefore, the focus of this section is on the results rather than the description of the scheme. A motivated reader is referred to [15]. The RHK-NOF is proved to be an $\eta$-NE action profile with $\eta \geqslant 1$. That is, any unilateral deviation from the RHKNOF by any of the transmitter-receiver pairs might lead to an individual rate improvement which is at most one bit per channel use. The description of the achievable $\eta$-Nash region $\underline{\mathcal{N}}_{\eta}$ is presented using the constants $a_{1, i}$; the functions $a_{2, i}:[0,1] \rightarrow \mathbb{R}_{+}, a_{l, i}:[0,1]^{2} \rightarrow \mathbb{R}_{+}$, with $l \in\{3, \ldots, 6\}$; and $a_{7, i}:[0,1]^{3} \rightarrow \mathbb{R}_{+}$, which are defined as follows, for all $i \in\{1,2\}$, with $j \in\{1,2\} \backslash\{i\}$ :

$$
\begin{aligned}
a_{1, i} & =\frac{1}{2} \log \left(2+\frac{\overrightarrow{\mathrm{SNR}_{i}}}{\mathrm{INR}_{j i}}\right)-\frac{1}{2}, \\
a_{2, i}(\rho) & =\frac{1}{2} \log \left(b_{1, i}(\rho)+1\right)-\frac{1}{2}, \\
a_{3, i}(\rho, \mu) & =\frac{1}{2} \log \left(\frac{\overleftarrow{\mathrm{SNR}}_{i}\left(b_{2, i}(\rho)+2\right)+b_{1, i}(1)+1}{\overleftarrow{\mathrm{SNR}}_{i}\left((1-\mu) b_{2, i}(\rho)+2\right)+b_{1, i}(1)+1}\right),
\end{aligned}
$$




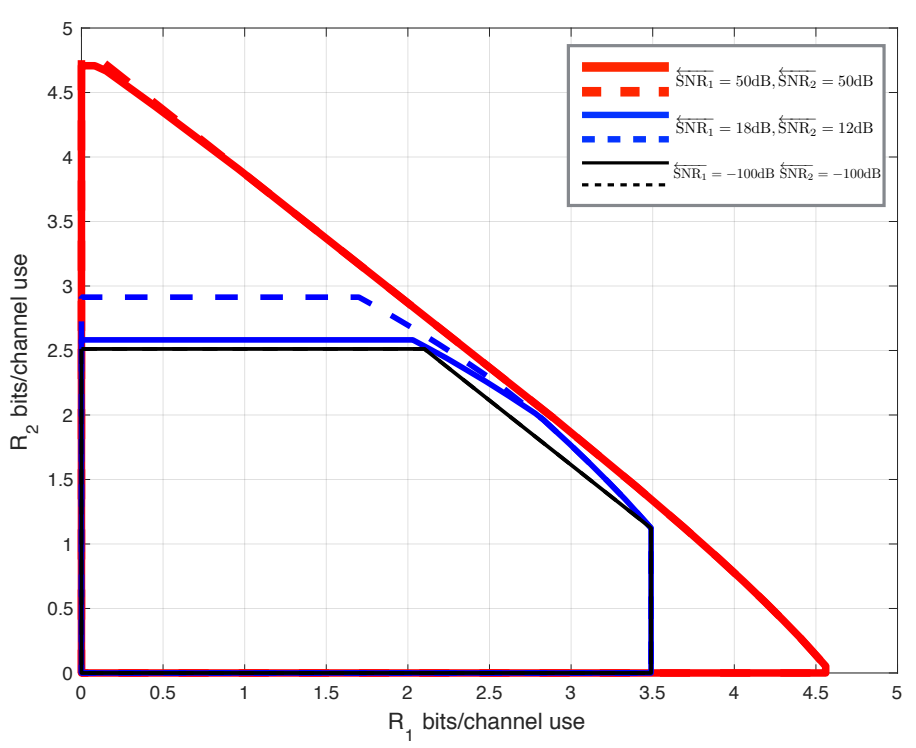

Fig. 4. Achievable capacity regions $\mathcal{C}$ (dashed-lines) in [4 Theorem 2] and achievable $\eta$-NE regions $\underline{\mathcal{N}}_{\eta}$ (solid lines) in Theorem 1 of the twouser GIC-NOF and two-user D-GIC-NOF with parameters $\mathrm{SNR}_{1}=24$ $\mathrm{dB}, \overrightarrow{\mathrm{SNR}}_{2}=18 \mathrm{~dB}, \mathrm{INR}_{12}=48 \mathrm{~dB}, \mathrm{INR}_{21}=30 \mathrm{~dB}, \overleftarrow{\mathrm{SNR}}_{1} \in$ $\{-100,18,50\} \mathrm{dB}, \overleftarrow{\mathrm{SNR}}_{2} \in\{-100,12,50\} \mathrm{dB}$ and $\eta=1$.

$$
\begin{aligned}
a_{4, i}(\rho, \mu)= & \frac{1}{2} \log \left((1-\mu) b_{2, i}(\rho)+2\right)-\frac{1}{2}, \\
a_{5, i}(\rho, \mu)= & \frac{1}{2} \log \left(2+\frac{\overrightarrow{\mathrm{SNR}}_{i}}{\mathrm{INR}_{j i}}+(1-\mu) b_{2, i}(\rho)\right)-\frac{1}{2}, \\
a_{6, i}(\rho, \mu)= & \frac{1}{2} \log \left(\frac{\overrightarrow{\mathrm{SNR}}_{i}}{\mathrm{INR}_{j i}}\left((1-\mu) b_{2, j}(\rho)+1\right)+2\right)-\frac{1}{2}, \\
a_{7, i}\left(\rho, \mu_{1}, \mu_{2}\right)= & \frac{1}{2} \log \left(\frac{\mathrm{SNR}_{i}}{\mathrm{INR}_{j i}}\left(\left(1-\mu_{i}\right) b_{2, j}(\rho)+1\right)\right. \\
& \left.+\left(1-\mu_{j}\right) b_{2, i}(\rho)+2\right)-\frac{1}{2},
\end{aligned}
$$

where the functions $b_{l, i}:[0,1] \rightarrow \mathbb{R}_{+}$, with $l \in\{1,2\}$ are defined as follows:

$$
\begin{aligned}
b_{1, i}(\rho) & ={\overrightarrow{\mathrm{SNR}_{i}}}_{i}+2 \rho \sqrt{\overrightarrow{\mathrm{SNR}}_{i} \mathrm{INR}_{i j}}+\mathrm{INR}_{i j} \text { and } \\
b_{2, i}(\rho) & =(1-\rho) \mathrm{INR}_{i j}-1
\end{aligned}
$$

Note that the functions in $(15)$ and $(16)$ depend on $\overrightarrow{S N R}_{1}$, $\overrightarrow{\mathrm{SNR}}_{2}, \mathrm{INR}_{12}, \mathrm{INR}_{21}, \mathrm{SNR}_{1}$, and $\mathrm{SNR}_{2}$, however as these parameters are fixed in this analysis, this dependence is not emphasized in the definition of these functions. Finally, using this notation, the achievable $\eta$-NE region is presented by Theorem 1 on the next page. The proof of Theorem 1 is presented in [8]. The inequalities in (17) are additional conditions to those defining the region $\mathcal{C}$ in [4, Theorem 2]. More specifically, the $\eta$-NE region is described by the intersection of the achievable region $\underline{\mathcal{C}}$ and the set of rate pairs $\left(R_{1}, R_{2}\right)$ satisfying (17).

Figure 2 shows the achievable region $\underline{\mathcal{C}}$ in [4, Theorem 2] of a two-user centralized GIC-NOF and the achievable $\eta$-NE region $\underline{\mathcal{N}}_{\eta}$ in Theorem 1 of a two-user D-GIC-NOF with parameters $\overrightarrow{\mathrm{SNR}}_{1}=24 \mathrm{~dB}, \overrightarrow{\mathrm{SNR}}_{2}=3 \mathrm{~dB}, \mathrm{INR}_{12}=16$ $\mathrm{dB}, \mathrm{INR}_{21}=9 \mathrm{~dB}, \widehat{\mathrm{SNR}}_{1} \in\{-100,18,50\} \mathrm{dB}, \mathrm{SNR}_{2} \in$ $\{-100,8,50\} \mathrm{dB}$ and $\eta=1$. Note that in this case, the feedback parameter $\overleftarrow{\mathrm{SNR}_{2}}$ does not have an effect on the achievable $\eta$-NE region $\underline{\mathcal{N}}_{\eta}$ and the achievable capacity region $\underline{\mathcal{C}}$ ([4, Theorem 2]). This is due to the fact that when one transmitter-receiver pair is in low interference regime (LIR) and the other transmitter-receiver pair is in high interference regime (HIR), feedback is useless on the transmitter-receiver pair in HIR [15], [16].

Figure 3 shows the achievable region $\mathcal{C}$ in [4, Theorem 2] of a two-user centralized GIC-NOF and the achievable $\eta$-NE region $\underline{\mathcal{N}}_{\eta}$ in Theorem 1 of a two-user D-GIC-NOF with parameters $\overrightarrow{\mathrm{SNR}}_{1}=24 \mathrm{~dB}, \overrightarrow{\mathrm{SNR}}_{2}=18 \mathrm{~dB}, \mathrm{INR}_{12}=16$ $\mathrm{dB}, \mathrm{INR}_{21}=10 \mathrm{~dB}, \overleftarrow{\mathrm{SNR}}_{1} \in\{-100,18,50\} \mathrm{dB}, \overleftarrow{\mathrm{SNR}}_{2} \in$ $\{-100,12,50\} \mathrm{dB}$ and $\eta=1$. Figure 4 shows the achievable region $\mathcal{C}$ in [4, Theorem 2] of a two-user centralized GIC$\mathrm{NOF}$ and the achievable $\eta$-NE region $\underline{\mathcal{N}}_{\eta}$ in Theorem 1 of a two-user D-GIC-NOF with parameters $\overrightarrow{\mathrm{SNR}}_{1}=24$ $\mathrm{dB}, \overrightarrow{\mathrm{SNR}}_{2}=18 \mathrm{~dB}, \mathrm{INR}_{12}=48 \mathrm{~dB}, \mathrm{INR}_{21}=30 \mathrm{~dB}$, $\overleftarrow{\mathrm{SNR}}_{1} \in\{-100,18,50\} \mathrm{dB}, \mathrm{SNR}_{2} \in\{-100,12,50\} \mathrm{dB}$ and $\eta=1$. In this case, the achievable $\eta$-NE region $\underline{\mathcal{N}}_{\eta}$ in Theorem 1 and achievable region $\underline{\mathcal{C}}$ on the capacity region [4, Theorem 2] are almost identical, which implies that in the cases in which $\overrightarrow{\mathrm{SNR}}_{i}<\mathrm{INR}_{i j}$, for both $i \in\{1,2\}$, with $j \in\{1,2\} \backslash\{i\}$, the achievable $\eta$-NE region is almost the same as the achievable capacity region in the centralized case studied in [4]. At low values of $\overleftarrow{S N R}_{1}$ and $\overleftarrow{\mathrm{SNR}}_{2}$, the achievable $\eta$-NE region approaches the rectangular region reported in [5] for the case of the two-user decentralized GIC (D-GIC). Alternatively, for high values of $\overleftarrow{\mathrm{SNR}}_{1}$ and $\overleftarrow{\mathrm{SNR}}_{2}$, the achievable $\eta$-NE region approaches the region reported in [6] for the case of the two-user decentralized GIC with perfect channel-output feedback (D-GIC-POF). These observations are formalized by the following corollaries.

Denote by $\underline{\mathcal{N}}_{\eta \mathrm{PF}}$ the achievable $\eta$-NE region of the twouser D-GIC-POF presented in [6]. The region $\underline{\mathcal{N}}_{\eta_{\mathrm{PF}}}$ can be obtained as a special case of Theorem 1 as shown by the following corollary.

Corollary 1 ( $\eta$-NE Region with Perfect Output Feedback): Let $\underline{\mathcal{N}}_{\eta \mathrm{PF}}$ denote the achievable $\eta$-NE region of the two-user D-GIC-POF with fixed parameters $\overrightarrow{\mathrm{SNR}}_{i}$ and $\mathrm{INR}_{i j}$, with $i \in\{1,2\}$ and $j \in\{1,2\} \backslash\{i\}$. Then, the following holds:

$$
\underline{\mathcal{N}}_{\eta_{\mathrm{PF}}}=\lim _{\substack{\overleftarrow{\mathrm{SNR}}_{1} \rightarrow \infty \\ \mathrm{SNR}_{2} \rightarrow \infty}} \frac{\mathcal{N}}{\overleftarrow{\mathrm{NR}}_{2}} \eta
$$

Denote by $\underline{\mathcal{N}}_{\eta \mathrm{WF}}$ the achievable $\eta$-NE region of the two-user D-GIC presented in [5]. The region $\underline{\mathcal{N}}_{\eta \mathrm{WF}}$ can be obtained as a special case of Theorem 1 as shown by the following corollary.

Corollary 2 ( $\eta$-NE Region without Output Feedback ): Let $\underline{\mathcal{N}}_{\eta \mathrm{WF}}$ denote the achievable $\eta-N E$ region of the two-user $D$ GIC, with fixed parameters $\overrightarrow{\mathrm{SNR}}_{i}$ and $\mathrm{INR}_{i j}$, with $i \in\{1,2\}$ 
Theorem 1: Let $\eta \geqslant 1$ be fixed. The achievable $\eta$-NE region $\underline{\mathcal{N}}_{\eta}$ is given by the closure of all possible achievable rate pairs $\left(R_{1}, R_{2}\right) \in \underline{\mathcal{C}}$ in [4. Theorem 2] that satisfy, for all $i \in\{1,2\}$ and $j \in\{1,2\} \backslash\{i\}$, the following conditions:

$$
\begin{aligned}
R_{i} \geqslant & \left(a_{2, i}(\rho)-a_{3, i}\left(\rho, \mu_{j}\right)-a_{4, i}\left(\rho, \mu_{j}\right)-\eta\right)^{+}, \\
R_{i} \leqslant & \min \left(a_{2, i}(\rho)+a_{3, j}\left(\rho, \mu_{i}\right)+a_{5, j}\left(\rho, \mu_{i}\right)-a_{2, j}(\rho)+\eta,\right. \\
& a_{3, i}\left(\rho, \mu_{j}\right)+a_{7, i}\left(\rho, \mu_{1}, \mu_{2}\right)+2 a_{3, j}\left(\rho, \mu_{i}\right)+a_{5, j}\left(\rho, \mu_{i}\right)-a_{2, j}(\rho)+\eta, \\
& \left.a_{2, i}(\rho)+a_{3, i}\left(\rho, \mu_{j}\right)+2 a_{3, j}\left(\rho, \mu_{i}\right)+a_{5, j}\left(\rho, \mu_{i}\right)+a_{7, j}\left(\rho, \mu_{1}, \mu_{2}\right)-2 a_{2, j}(\rho)+2 \eta\right), \\
R_{1}+R_{2} \leqslant & a_{1, i}+a_{3, i}\left(\rho, \mu_{j}\right)+a_{7, i}\left(\rho, \mu_{1}, \mu_{2}\right)+a_{2, j}(\rho)+a_{3, j}\left(\rho, \mu_{1}\right)-a_{2, i}(\rho)+\eta,
\end{aligned}
$$

for all $\left(\rho, \mu_{1}, \mu_{2}\right) \in\left[0,\left(1-\max \left(\frac{1}{\mathrm{INR}_{12}}, \frac{1}{\mathrm{INR}_{21}}\right)\right)^{+}\right] \times[0,1] \times[0,1]$.

and $j \in\{1,2\} \backslash\{i\}$. Then, the following holds:

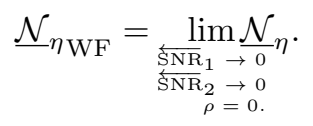

\section{B. Imposibility Region}

This section introduces an imposibility region, denoted by $\overline{\mathcal{N}}_{\eta}$. That is, $\overline{\mathcal{N}}_{\eta} \supseteq \mathcal{N}_{\eta}$. More specifically, any rate pair $\left(R_{1}, R_{2}\right) \in \overline{\mathcal{N}}_{\eta}^{\mathrm{c}}$ is not an $\eta$-NE. This region is described in terms of the convex region $\overline{\mathcal{B}}_{\mathrm{G}-\mathrm{IC}-\mathrm{NOF}}$. Here, for the case of the two-user D-GIC-NOF, the region $\overline{\mathcal{B}}_{\mathrm{G}-\mathrm{IC}-\mathrm{NOF}}$ is given by the closure of the rate pairs $\left(R_{1}, R_{2}\right) \in \mathbb{R}_{+}^{2}$ that satisfy for all $i \in\{1,2\}$, with $j \in\{1,2\} \backslash\{i\}$ :

$$
\begin{aligned}
\overline{\mathcal{B}}_{\mathrm{G}-\mathrm{IC}-\mathrm{NOF}}= & \left\{\left(R_{1}, R_{2}\right) \in \mathbb{R}_{+}^{2}: R_{i} \geqslant L_{i},\right. \\
& \text { for all } i \in \mathcal{K}=\{1,2\}\},
\end{aligned}
$$

where,

$$
L_{i} \triangleq\left(\frac{1}{2} \log \left(1+\frac{\overrightarrow{\mathrm{SNR}}_{i}}{1+\mathrm{INR}_{i j}}\right)-\eta\right)^{+} .
$$

Note that $L_{i}$ is the rate achieved by the transmitter-receiver pair $i$ when it saturates the power constraint in (5) and treats interference as noise. Following this notation, the imposibility region of the two-user GIC-NOF, i.e., $\overline{\mathcal{N}}_{\eta}$, can be described as follows.

Theorem 2: Let $\eta \geqslant 1$ be fixed. The imposibility region $\overline{\mathcal{N}}_{\eta}$ of the two-user D-GIC-NOF is given by the closure of all possible non-negative rate pairs $\left(R_{1}, R_{2}\right) \in \overline{\mathcal{C}} \cap \overline{\mathcal{B}}_{\mathrm{G}-\mathrm{IC}-\mathrm{NOF}}$ for all $\rho \in[0,1]$.

The impossibility region in Theorem 2 has been first presented in [6] and it is very loose in this case. A better impossibility region is presented in [15].

\section{CONCLUSIONS}

In this paper, an achievable $\eta$-Nash equilibrium ( $\eta$-NE) region for the two-user Gaussian interference channel with noisy channel-output feedback has been presented for all $\eta \geqslant 1$. This result generalizes the existing achievable regions of the $\eta$-NE for the the cases without feedback and with perfect channel-output feedback.

\section{REFERENCES}

[1] R. H. Etkin, D. N. C. Tse, and W. Hua, "Gaussian interference channel capacity to within one bit," IEEE Trans. Inf. Theory, vol. 54, no. 12, pp. 5534-5562, Dec. 2008.

[2] C. Suh and D. N. C. Tse, "Feedback capacity of the Gaussian interference channel to within 2 bits," IEEE Trans. Inf. Theory, vol. 57, no. 5, pp. 2667-2685, May. 2011.

[3] S.-Q. Le, R. Tandon, M. Motani, and H. V. Poor, "Approximate capacity region for the symmetric Gaussian interference channel with noisy feedback," IEEE Trans. Inf. Theory, vol. 61, no. 7, pp. 3737-3762, Jul. 2015.

[4] V. Quintero, S. M. Perlaza, I. Esnaola, and J.-M. Gorce, "Approximate capacity region of the two-user Gaussian interference channel with noisy channel-output feedback," IEEE Trans. Inf. Theory, vol. 64, no. 7, pp. 5326-5358, Jul 2018.

[5] R. A. Berry and D. N. C. Tse, "Shannon meets Nash on the interference channel," IEEE Trans. Inf. Theory, vol. 57, no. 5, pp. 2821-2836, May. 2011.

[6] S. M. Perlaza, R. Tandon, H. V. Poor, and Z. Han, "Perfect output feedback in the two-user decentralized interference channel," IEEE Trans. Inf. Theory, vol. 61, no. 10, pp. 5441-5462, Oct. 2015.

[7] V. Quintero, S. M. Perlaza, J.-M. Gorce, and H. V. Poor, "Nash region of the linear deterministic interference channel with noisy output feedback," in Proc. IEEE Int. Symp. on Inform. Theory (ISIT), Aachen, Germany, Jun. 2017.

[8] _ "Decentralized interference channels with noisy output feedback," INRIA, Lyon, France, Tech. Rep. 9011, Jan. 2017.

[9] R. D. Yates, D. Tse, and Z. Li, "Secret communication on interference channels," in Proc. IEEE International Symposium on Information Theory (ISIT), Toronto, Canada, Jul. 2008.

[10] R. Berry and D. N. C. Tse, "Information theoretic games on interference channels," in Proc. IEEE International Symposium on Information Theory (ISIT), Toronto,Canada, Jul. 2008.

[11] J. F. Nash, "Equilibrium points in n-person games," Proc. National Academy of Sciences of the United States of America, vol. 36, no. 1, pp. 48-49, Jan. 1950.

[12] T. S. Han and K. Kobayashi, "A new achievable rate region for the interference channel," IEEE Trans. Inf. Theory, vol. 27, no. 1, pp. 4960, Jan. 1981.

[13] T. M. Cover and C. S. K. Leung, "An achievable rate region for the multiple-access channel with feedback," IEEE Trans. Inf. Theory, vol. 27, no. 3, pp. 292-298, May. 1981.

[14] F. M. J. Willems, "Information theoretical results for multiple access channels," Ph.D. dissertation, Katholieke Universiteit, Leuven, Belgium, Oct. 1982.

[15] V. Quintero, "Noisy channel-output feedback in the interference channel," Ph.D. dissertation, Université de Lyon, Lyon, France., Dec. 2017.

[16] V. Quintero, S. M. Perlaza, I. Esnaola, and J.-M. Gorce, "When does output feedback enlarge the capacity of the interference channel?" IEEE Trans. Commun., vol. 66, no. 2, pp. 615-628, Feb. 2018. 